\title{
Solvability of a two-point fractional boundary value problem
}

\author{
Assia Guezane-Lakoud ${ }^{a, *}$, Rabah Khaldi ${ }^{\text {b }}$ \\ ${ }^{a}$ Laboratory of Advanced Materials. Faculty of Sciences, Badji Mokhtar-Annaba University, P. O. Box 12, 23000 Annaba, Algeria. \\ ${ }^{b}$ Laboratory LASEA. Faculty of Sciences, Badji Mokhtar-Annaba University, P. O. Box 12, 23000 Annaba, Algeria. \\ This paper is dedicated to Professor Ljubomir Ćirić \\ Communicated by Professor V. Berinde
}

\begin{abstract}
The aim of this paper is the study of the existence and uniqueness of solutions for a two-point fractional boundary value problem, by means of Banach contraction principle and Leray Schauder nonlinear alternative. Some examples are given.(C)2012 NGA. All rights reserved.
\end{abstract}

Keywords: Fractional Caputo derivative, Banach Contraction principle, Leray Schauder nonlinear alternative.

2010 MSC: Primary 34B10; Secondary 26A33, 34B15.

\section{Introduction and Preliminaries}

Motived by the big numbers of applications of fractional differential equations in various fields of science and engineering such as control, porous media, electrochemistry, viscoelasticity, electromagnetic, and other fields, see $[1,5,10,11]$, many fractional boundary value problems with the help of technics of nonlinear analysis, have been investigated in the last few decades.

This paper deals with the existence and uniqueness of solutions for the boundary value problem (P1):

$$
\begin{gathered}
{ }^{c} D_{0^{+}}^{q} u(t)=f\left(t, u(t),{ }^{c} D_{0^{+}}^{\sigma} u(t)\right), \quad 0<t<1 \\
u(0)=u(1)=u^{\prime \prime}(1)=u^{\prime \prime}(0)=0,
\end{gathered}
$$

\footnotetext{
${ }^{*}$ Corresponding author

Email addresses: a_guezane@yahoo.fr (Assia Guezane-Lakoud ), rkhadi@yahoo.fr (Rabah Khaldi)
} 
where $f:[0,1] \times \mathbb{R} \times \mathbb{R} \rightarrow \mathbb{R}$ is a given function, $3<q<4,1<\sigma<2$ and ${ }^{c} D_{0^{+}}^{q}$ denotes the Caputo's fractional derivative. It is Shown that the Caputo's fractional derivative, allows the utilization of physically interpretation of boundary conditions. For more details on the geometric and physical interpretation for Caputo fractional derivatives types, see [11]. Our results allow the function $f$ to depend on the fractional derivative ${ }^{c} D_{0^{+}}^{\sigma} u(t)$ which leads to some difficulties. By using Banach contraction principle and Leray Schauder nonlinear alternative, the existence and uniqueness of solution for the fractional boundary value problem (P1) are investigated. No contributions exist, as far as we know, concerning the existence of solutions of the fractional differential equation (1.1) jointly with the nonlocal condition (1.2).

Most of papers dealing with similar problems are devoted to the solvability of nonlinear fractional differential equation or to the existence and multiplicity of positive solutions $[2,6,8,9,12,13-16]$. Moreover, in [9], Sihua Liang and Jihui Zhang considered the following nonlinear fractional boundary value problem

$$
\begin{array}{r}
D_{0^{+}}^{q} u(t)+f(t, u(t))=0, \quad 0<t<1 \\
u(0)=u^{\prime}(0)=u^{\prime \prime}(0)=u^{\prime \prime}(1)=0
\end{array}
$$

where $3<q \leq 4$ and $D_{0^{+}}^{q}$ is the Riemann-Liouville fractional derivative. Using lower and upper solution method and fixed-point theorems, some results on the existence of positive solutions are obtained.

With the help of Leray-Schauder nonlinear alternative, a fixed-point theorem on cones and a mixed monotone method, $\mathrm{Xu}$, Jiang and Yuan [14] have established the positivity of multiple solutions to the following fractional differential equation,

$$
\begin{array}{r}
D_{0^{+}}^{q} u(t)+f(t, u(t))=0, \quad 0<t<1 \\
u(0)=u(1)=u^{\prime}(0)=u^{\prime}(1)=0
\end{array}
$$

where $3<q \leq 4$ and $D_{0^{+}}^{q}$ is the Riemann-Liouville fractional derivative.

In [2], Bai established the existence of triple positive solutions for the nonlinear fractional differential equation boundary value problem:

$$
\begin{array}{r}
D_{0^{+}}^{q} u(t)+a(t) f\left(t, u(t), u^{\prime}(t)\right)=0, \quad 0<t<1 \\
u(0)=u^{\prime}(0)=u^{\prime \prime}(0)=u^{\prime \prime}(1)=0,
\end{array}
$$

where $3<q \leq 4$ and $D_{0^{+}}^{q}$ is the Riemann-Liouville fractional derivative. This paper is organized as follows, in the next section we cite some Definitions and Lemmas needed in our proofs. Section 3 treats the existence and uniqueness of solution by using Banach contraction principle and Leray-Schauder nonlinear alternative. Some examples are given illustrating the previous results.

\section{Preliminaries and Lemmas}

In this section, we introduce definitions and preliminary facts that are used throughout this paper.

Definition 2.1. If $g \in C([a, b])$ and $\alpha>0$, then the Riemann-Liouville fractional integral is defined by $I_{a^{+}}^{\alpha} g(t)=\frac{1}{\Gamma(\alpha)} \int_{a}^{t} \frac{g(s)}{(t-s)^{1-\alpha}} d s$.

Definition 2.2. Let $\alpha \geqq 0, n=[\alpha]+1$. If $f \in A C^{n}[a, b]$ then the Caputo fractional derivative of order $\alpha$ of $f$ defined by ${ }^{c} D_{a^{+}}^{\alpha} g(t)=\frac{1}{\Gamma(n-\alpha)} \int_{a}^{t} \frac{g^{(n)}(s)}{(t-s)^{\alpha-n+1}} d s$, exist almost everywhere on $[a, b]([\alpha]$ is the entire part of $\alpha$ ). 
Lemma 2.3. [7] Let $\alpha, \beta>0$ and $n=[\alpha]+1$, then the following relations hold: ${ }^{c} D_{0^{+}}^{\alpha} t^{\beta-1}=\frac{\Gamma(\beta)}{\Gamma(\beta-\alpha)} t^{\beta-\alpha-1}$, $\beta>n$ and ${ }^{c} D_{0^{+}}^{\alpha} t^{k}=0, k=0,1,2, \ldots, n-1$.

Lemma 2.4. [7] For $\alpha>0, g(t) \in C(0,1)$, the homogenous fractional differential equation ${ }^{c} D_{a^{+}}^{\alpha} g(t)=0$ has a solution $g(t)=c_{1}+c_{2} t+c_{3} t^{2}+\ldots+c_{n} t^{n-1}$, where, $c_{i} \in R, i=0, \ldots, n$, and $n=[\alpha]+1$, ( $\alpha$ non-integer)

Denote by $L^{1}([0,1], \mathbb{R})$ the Banach space of Lebesgue integrable functions from $[0,1]$ into $\mathbb{R}$ with the norm $\|y\|_{L^{1}}=\int_{0}^{1}|y(t)| d t$.

The following Lemmas gives some properties of Riemann-Liouville fractional integrals and Caputo fractional derivative.

Lemma 2.5. [1] Let $p, q \geq 0, f \in L_{1}[a, b]$. Then $I_{0^{+}}^{p} I_{0^{+}}^{q} f(t)=I_{0^{+}}^{p+q} f(t)=I_{0^{+}}^{q} I_{0^{+}}^{p} f(t)$ and ${ }^{c} D_{0^{+}}^{q} I_{0^{+}}^{q} f(t)=$ $f(t)$, for all $t \in[a, b]$.

Lemma 2.6. [7] Let $\beta>\alpha>0$. Then the formula ${ }^{c} D_{0^{+}}^{\alpha} I_{0^{+}}^{\beta} f(t)=I_{0^{+}}^{\beta-\alpha} f(t)$, holds almost everywhere on $t \in[a, b]$, for $f \in L_{1}[a, b]$ and it is valid at any point $x \in[a, b]$ if $f \in C[a, b]$.

Now we start by solving an auxiliary problem.

Lemma 2.7. Let $3<q<4,1<\sigma<2$ and $y \in C[0,1]$. The unique solution of the fractional boundary value problem

$$
\left\{\begin{array}{c}
{ }^{c} D_{0^{+}}^{q} u(t)=y(t), 0<t<1 \\
u(0)=u(1)=u^{\prime \prime}(1)=u^{\prime \prime}(0)=0,
\end{array}\right.
$$

is given by

$$
u(t)=\frac{1}{\Gamma(q)} \int_{0}^{1} G(t, s) y(s) d s
$$

where

$$
G(t, s)=\left\{\begin{array}{c}
(t-s)^{q-1}-t(1-s)^{q-1}+\frac{q(q-1)}{6} t\left(1-t^{2}\right)(1-s)^{q-3}, \quad s \leq t \\
-t(1-s)^{q-1}+\frac{q(q-1)}{6} t\left(1-t^{2}\right)(1-s)^{q-3}, \quad t \leq s .
\end{array}\right.
$$

Proof. In view of Lemmas 2.4 and 2.5 the equation (2.1) is equivalent to the integral equation

$$
u(t)=I_{0^{+}}^{q} y(t)+c_{1}+c_{2} t+c_{3} t^{2}+c_{4} t^{3}
$$

for some $c_{i} \in \mathbb{R}$. The boundary condition $u(0)=0$ gives $c_{1}=0$ and from the boundary condition $u(1)=0$ we conclude $I_{0^{+}}^{q} y(1)+c_{2}+c_{3}+c_{4}=0$. Differentiating twice both sides of (2.4) and using Lemma 2.6 it yields $u^{\prime \prime}(t)=I_{0^{+}}^{q-2} y(t)+2 c_{3}+6 c_{4} t$. The boundary condition $u^{\prime \prime}(0)=0$ implies $c_{3}=0$ and from $u^{\prime \prime}(1)=0$ we deduce that $c_{4}=\frac{-I_{0^{+}}^{q-2} y(1)}{6}$, hence $\frac{I_{0^{+}}^{q-2} y(1)}{6}-I_{0^{+}}^{q} y(1)=c_{2}$. Substituting $c_{i}, i=1, \ldots 4$, by their values in (2.4), we get

$$
u(t)=I_{0^{+}}^{q} y(t)+t\left(\frac{I_{0^{+}}^{q-2} y(1)}{6}-I_{0^{+}}^{q} y(1)\right)-\frac{t^{3} I_{0^{+}}^{q-2} y(1)}{6}
$$

that can be written as

$$
\begin{aligned}
& u(t)= \frac{1}{\Gamma(q)} \int_{0}^{t}\left((t-s)^{q-1}-t(1-s)^{q-1}+\right. \\
&\left.\frac{q(q-1) t\left(1-t^{2}\right)(1-s)^{q-3}}{6}\right) y(s) d s \\
&+\frac{1}{\Gamma(q)} \int_{t}^{1}\left[-t(1-s)^{q-1}+\frac{q(q-1)}{6} t\left(1-t^{2}\right)(1-s)^{q-3}\right] y(s) d s
\end{aligned}
$$

that is equivalent to $u(t)=\frac{1}{\Gamma(q)} \int_{0}^{1} G(t, s) y(s) d s$ where $G$ is defined by (2.3). The proof is complete. 


\section{Existence and Uniqueness results}

In this section we prove the existence and uniqueness of solution in the Banach space $E$ defined by all functions $u \in C^{2}([0,1], \mathbb{R})$, equipped with the norm $\|u\|=\max _{t \in[0,1]}|u(t)|+\max _{t \in[0,1]}\left|{ }^{c} D_{0^{+}}^{\sigma} u(t)\right|$. We know that for $1<\sigma<2$ we have ${ }^{c} D_{0^{+}}^{\sigma} u \in C[0,1]$ see [7]. Throughout this section, we suppose that $f \in$ $C([0,1] \times \mathbb{R} \times \mathbb{R}, \mathbb{R})$. Define the integral operator $T: E \rightarrow E$ by $T u(t)=\frac{1}{\Gamma(q)} \int_{0}^{1} G(t, s) f\left(s, u(s),{ }^{c} D_{0^{+}}^{\sigma} u(s)\right) d s$.

Lemma 3.1. The function $u \in E$ is solution of the fractional boundary value problem (P1) if and only if $T u(t)=u(t), \forall t \in[0,1]$.

Proof. Suppose that $u$ is solution of (P1) and let $v(t)=\frac{1}{\Gamma(q)} \int_{0}^{1} G(t, s) f\left(s, u(s),{ }^{c} D_{0^{+}}^{\sigma} u(s)\right) d s$. In view of $(2.5)$ we have

$$
\begin{aligned}
v(t)= & I_{0^{+}}^{q} f\left(t, u(t),{ }^{c} D_{0^{+}}^{\sigma} u(t)\right)- \\
& t I_{0^{+}}^{q} f\left(1, u(1),{ }^{c} D_{0^{+}}^{\sigma} u(1)\right)+\frac{t\left(1-t^{2}\right)}{6} I_{0^{+}}^{q-2} f\left(1, u(1),{ }^{c} D_{0^{+}}^{\sigma} u(1)\right) .
\end{aligned}
$$

with the help of Lemmas 2.6 and 2.3, we obtain

$$
\begin{aligned}
{ }^{c} D_{0^{+}}^{q} v(t)= & { }^{c} D_{0^{+}}^{q} I_{0^{+}}^{q} f\left(t, u(t),{ }^{c} D_{0^{+}}^{\sigma} u(t)\right)-\left({ }^{c} D_{0^{+}}^{q} t\right) I_{0^{+}}^{q} f\left(1, u(1),{ }^{c} D_{0^{+}}^{\sigma} u(1)\right) \\
& +\frac{\left({ }^{c} D_{0^{+}}^{q} t\left(1-t^{2}\right)\right)}{6} I_{0^{+}}^{q-2} f\left(1, u(1),{ }^{c} D_{0^{+}}^{\sigma} u(1)\right) \\
= & f\left(t, u(t),{ }^{c} D_{0^{+}}^{\sigma} u(t)\right) .
\end{aligned}
$$

It is clear that $v$ satisfies conditions (1.2), then it is a solution for the problem (P1). The proof is complete.

Theorem 3.2. Assume that there exists nonnegative functions $g, h \in L^{1}\left([0,1], \mathbb{R}_{+}\right)$such that for all $x, y, \bar{x}, \bar{y} \in \mathbb{R}$ and $t \in[0,1]$, we have

$$
|f(t, x, \bar{x})-f(t, y, \bar{y})| \leq g(t)|x-y|+h(t)|\bar{x}-\bar{y}|,
$$

and

$$
C_{g}+C_{h}<\frac{1}{2}, \quad A_{g}+A_{h}<\frac{1}{2}
$$

where

$$
\begin{aligned}
C_{g} & =\left(\left\|I_{0^{+}}^{q-1} g\right\|_{L^{1}}+I_{0^{+}}^{q} g(1)+I_{0^{+}}^{q-2} g(1)\right), \\
A_{g} & =\left\|I_{0^{+}}^{q-\sigma-1} g\right\|_{L^{1}}+\frac{I_{0^{+}}^{q-2} g(1)}{\Gamma(4-\sigma)}, \\
C_{h} & =\left(\left\|I_{0^{+}}^{q-1} h\right\|_{L^{1}}+I_{0^{+}}^{q} h(1)+I_{0^{+}}^{q-2} h(1)\right), \\
A_{h} & =\left\|I_{0^{+}}^{q-\sigma-1} h\right\|_{L^{1}}+\frac{I_{0^{+}}^{q-2} h(1)}{\Gamma(4-\sigma)} .
\end{aligned}
$$

Then the fractional boundary value problem(P1) has a unique solution $u$ in E.

To prove Theorem 3.2, we use the following properties of Riemann-Liouville fractional integrals.

Lemma 3.3. Let $q>0, f \in L_{1}\left([a, b], \mathbb{R}_{+}\right)$. Then, for all $t \in[a, b]$ we have $I_{0^{+}}^{q+1} f(t) \leq\left\|I_{0^{+}}^{q} f\right\|_{L^{1}}$. 
Proof. Let $f \in L_{1}\left([a, b], \mathbb{R}_{+}\right)$, then

$$
\begin{aligned}
\left\|I_{0^{+}}^{q} f\right\|_{L^{1}} & =\int_{0}^{1} I_{0^{+}}^{q} f(r) d r \geq \frac{1}{\Gamma(q)} \int_{0}^{t} \int_{0}^{r} \frac{f(s)}{(r-s)^{1-q}} d s d r \\
& =\frac{1}{\Gamma(q)} \int_{0}^{t}\left(\int_{s}^{t} \frac{f(s)}{(r-s)^{1-q}} d r\right) d s=I_{0^{+}}^{q+1} f(t) .
\end{aligned}
$$

Now we prove Theorem 3.2.

Proof. We shall prove that $T$ is a contraction. In fact, for any $u, v \in E$, we have

$$
\begin{aligned}
& T u(t)-T v(t) \\
= & \frac{1}{\Gamma(q)} \int_{0}^{1} G(t, s)\left(f\left(s, u(s),{ }^{c} D_{0^{+}}^{\sigma} u(s)\right)-f\left(s, v(s),{ }^{c} D_{0^{+}}^{\sigma} v(s)\right)\right) d s \\
= & I_{0^{+}}^{q}\left(f\left(t, u(t),{ }^{c} D_{0^{+}}^{\sigma} u(t)\right)-f\left(s, v(t),{ }^{c} D_{0^{+}}^{\sigma} v(t)\right)\right) \\
& -t\left[I_{0^{+}}^{q}\left(f\left(1, u(1),{ }^{c} D_{0^{+}}^{\sigma} u(1)\right)-f\left(1, v(1),{ }^{c} D_{0^{+}}^{\sigma} v(1)\right)\right)\right. \\
& \left.+\frac{t\left(1-t^{2}\right)}{6} I_{0^{+}}^{q-2}\left(f\left(1, u(1),{ }^{c} D_{0^{+}}^{\sigma} u(1)\right)-f\left(1, v(1),{ }^{c} D_{0^{+}}^{\sigma} v(1)\right)\right)\right] .
\end{aligned}
$$

Inequality (3.1) implies that

$$
\begin{aligned}
& |T u(t)-T v(t)| \leq \max |u(t)-v(t)|\left(I_{0^{+}}^{q} g(t)+t I_{0^{+}}^{q} g(1)+\frac{t\left(1-t^{2}\right)}{6} I_{0^{+}}^{q-2} g(1)\right) \\
& \quad+\max \left|{ }^{c} D_{0^{+}}^{\sigma} u(t)-{ }^{c} D_{0^{+}}^{\sigma} v(t)\right|\left(I_{0^{+}}^{q} h(t)+t I_{0^{+}}^{q} h(1)+\frac{t\left(1-t^{2}\right)}{6} I_{0^{+}}^{q-2} h(1)\right) .
\end{aligned}
$$

From Lemma 3.3 we deduce

$$
\begin{aligned}
& |T u(t)-T v(t)| \leq\|u-v\|\left[\left\|I_{0^{+}}^{q-1} g\right\|_{L^{1}}+I_{0^{+}}^{q} g(1)+\frac{\sqrt{3}}{27} I_{0^{+}}^{q-2} g(1)\right. \\
& \left.+\left\|I_{0^{+}}^{q-1} h\right\|_{L^{1}}+I_{0^{+}}^{q} h(1)+\frac{\sqrt{3}}{27} I_{0^{+}}^{q-2} h(1)\right] \leq\|u-v\|\left(C_{g}+C_{h}\right),
\end{aligned}
$$

in view of (3.2) it yields

$$
|T u-T v|<\frac{\|u-v\|}{2} .
$$

On the other hand we have

$$
\begin{gathered}
{ }^{c} D_{0^{+}}^{\sigma} T u-{ }^{c} D_{0^{+}}^{\sigma} T v=I_{0^{+}}^{q-\sigma}\left(f\left(t, u(t),{ }^{c} D_{0^{+}}^{\sigma} u(t)\right)-f\left(s, v(t),{ }^{c} D_{0^{+}}^{\sigma} v(t)\right)\right) \\
\left.-\frac{t^{3-\sigma}}{\Gamma(4-\sigma)} I_{0^{+}}^{q-2}\left(f\left(1, u(1),{ }^{c} D_{0^{+}}^{\sigma} u(1)\right)-f\left(1, v(1),{ }^{c} D_{0^{+}}^{\sigma} v(1)\right)\right)\right] .
\end{gathered}
$$

Consequently (3.4) becomes

$$
\begin{aligned}
& \left|{ }^{c} D_{0^{+}}^{\sigma} T u-{ }^{c} D_{0^{+}}^{\sigma} T v\right| \\
\leq & \|u-v\|\left[\left\|I_{0^{+}}^{q-\sigma-1} g\right\|_{L^{1}}+\frac{I_{0^{+}}^{q-2} g(1)}{\Gamma(4-\sigma)}+\left\|I_{0^{+}}^{q-\sigma-1} h\right\|_{L^{1}}+\frac{I_{0^{+}}^{q-2} h(1)}{\Gamma(4-\sigma)}\right]
\end{aligned}
$$


Then

$$
\left|{ }^{c} D_{0^{+}}^{\sigma} T u-{ }^{c} D_{0^{+}}^{\sigma} T v\right| \leq\|u-v\|\left(A_{g}+A_{h}\right) .
$$

With the help of hypothesis (3.2) it yields

$$
\left|{ }^{c} D_{0^{+}}^{\sigma} T u-{ }^{c} D_{0^{+}}^{\sigma} T v\right|<\frac{\|u-v\|}{2} .
$$

Summing (3.3) and (3.6) we deduce $\|T u-T v\|<\|u-v\|$, from here, the Banach contraction mapping principle ensures the uniqueness of solution for the fractional boundary value problem (P1). This finishes the proof.

Now we give an existence result for the fractional boundary value problem (P1).

Theorem 3.4. Assume that $f(t, 0,0) \neq 0$ and there exists nonnegative functions $k, h, g \in L^{1}\left([0,1], \mathbb{R}_{+}\right)$, $\phi, \psi \in C\left(\mathbb{R}_{+}, \mathbb{R}_{+}^{*}\right)$ nondecreasing on $\mathbb{R}_{+}$and $r>0$, such that

$$
\begin{gathered}
|f(t, x, \bar{x})| \leq k(t) \psi(|x|)+h(t) \phi(|\bar{x}|)+g(t), \\
(\psi(r)+\phi(r)+1)\left(\frac{C_{1}}{\Gamma(q)}+C_{2}\right)<r,
\end{gathered}
$$

where $C_{1}=\max \left\{C_{k}, C_{h}, C_{g}\right\}, C_{2}=\max \left\{A_{k}, A_{h}, A_{g}\right\}, C_{h}$ and $C_{g}$ are defined as in Theorem 3.2 and

$$
\begin{aligned}
C_{k} & =\left\|I_{0^{+}}^{q-1} k\right\|_{L^{1}}+I_{0^{+}}^{q} k(1)+I_{0^{+}}^{q-2} k(1), \\
A_{k} & =\left\|I_{0^{+}}^{q-\sigma-1} k\right\|_{L^{1}}+\frac{I_{0^{+}}^{q-2} k(1)}{\Gamma(4-\sigma)} .
\end{aligned}
$$

Then the fractional boundary value problem (P1) has at least one nontrivial solution $u^{*} \in E$.

To prove this Theorem, we apply Leray-Schauder nonlinear alternative:

Lemma 3.5. [4]. Let $F$ be a Banach space and $\Omega$ a bounded open subset of $F, 0 \in \Omega$. $T: \bar{\Omega} \rightarrow F$ be a completely continuous operator. Then, either there exists $x \in \partial \Omega, \lambda>1$ such that $T(x)=\lambda x$, or there exists a fixed point $x^{*} \in \bar{\Omega}$.

Proof. First let us prove that $T$ is completely continuous. It is clear that $T$ is continuous since $f$ and $G$ are continuous. Let $B_{r}=\{u \in E,\|u\| \leq r\}$ be a bounded subset in $E$. We shall prove that $T\left(B_{r}\right)$ is relatively compact.

i) We will show that $T$ maps bounded sets into bounded sets in $E$. In fact, it suffices to show that for any $u \in B_{r}$ there exists a positive constant $C$ such that $\|T u\| \leq C$. Using (3.7) we have

$$
|T u(t)| \leq \frac{1}{\Gamma(q)} \int_{0}^{1}|G(t, s)|\left[k(s) \psi(|u(s)|)+h(s) \phi\left(\mid{ }^{c} D_{0^{+}}^{\sigma} u(s \mid)+g(s)\right] d s .\right.
$$

Since $\psi$ and $\phi$ are nondecreasing then (3.9) implies

$$
\begin{aligned}
|T u(t)| & \leq \frac{1}{\Gamma(q)} \int_{0}^{1}|G(t, s)|[k(s) \psi(\|u\|)+h(s) \phi(\|u\|)+g(s)] d s \\
& \leq \frac{1}{\Gamma(q)} \int_{0}^{1}|G(t, s)|[k(s) \psi(r)+h(s) \phi(r)+g(s)] d s,
\end{aligned}
$$


using similar technics as to get (3.3) it yields

$$
\begin{aligned}
|T u(t)| \leq & \frac{1}{\Gamma(q)}\left[\psi(r)\left(\left\|I_{0^{+}}^{q-1} k\right\|_{L^{1}}+I_{0^{+}}^{q} k(1)+I_{0^{+}}^{q-2} k(1)\right)\right. \\
& +\phi(r)\left(\left\|I_{0^{+}}^{q-1} h\right\|_{L^{1}}+I_{0^{+}}^{q} h(1)+I_{0^{+}}^{q-2} h(1)\right) \\
& \left.+\left(\left\|I_{0^{+}}^{q-1} g\right\|_{L^{1}}+I_{0^{+}}^{q} g(1)+I_{0^{+}}^{q-2} g(1)\right)\right] \\
& =\frac{1}{\Gamma(q)}\left(C_{k} \psi(r)+C_{h} \phi(r)+C_{g}\right) .
\end{aligned}
$$

Hence

$$
|T u(t)| \leq \frac{C_{1}}{\Gamma(q)}[\psi(r)+\phi(r)+1]
$$

Moreover, we have

$$
\begin{gathered}
\left|{ }^{c} D_{0^{+}}^{\sigma} T u\right| \leq \psi(r)\left(\left\|I_{0^{+}}^{q-\sigma-1} k\right\|_{L^{1}}+\frac{I_{0^{+}}^{q-2} k(1)}{\Gamma(4-\sigma)}\right) \\
+\phi(r)\left(\left\|I_{0^{+}}^{q-\sigma-1} h\right\|_{L^{1}}+\frac{I_{0^{+}}^{q-2} h(1)}{\Gamma(4-\sigma)}\right)+\left\|I_{0^{+}}^{q-\sigma-1} g\right\|_{L^{1}}+\frac{I_{0^{+}}^{q-2} g(1)}{\Gamma(4-\sigma)} .
\end{gathered}
$$

Using (3.5) we obtain

$$
\left|{ }^{c} D_{0^{+}}^{\sigma} T u\right| \leq C_{2}(\psi(r)+\phi(r)+1) .
$$

From (3.11) and (3.13) we get

$$
\|T u\|=(\psi(r)+\phi(r)+1)\left(\frac{C_{1}}{\Gamma(q)}+C_{2}\right)=C,
$$

then $T\left(B_{r}\right)$ is uniformly bounded.

ii) We will show that $T$ maps bounded sets into equicontinuous sets of $E$. Indeed for any $t_{1}, t_{2} \in[0,1]$, $t_{1}<t_{2}$ and $u \in B_{r}$, let $C^{\prime}=\max \left(\left|f\left(t, u(t),{ }^{c} D_{0^{+}}^{\sigma} u(t)\right)\right|, 0 \leq t \leq 1,\|u\|<r\right)$, therefore

$$
\begin{gathered}
\left|T u\left(t_{1}\right)-T u\left(t_{2}\right)\right| \leq \frac{C^{\prime}}{\Gamma(q)}\left(\int_{0}^{t_{1}}\left|G\left(t_{1}, s\right)-G\left(t_{2}, s\right)\right| d s+\right. \\
\left.\int_{t_{1}}^{t_{2}}\left|G\left(t_{1}, s\right)-G\left(t_{2}, s\right)\right| d s+\int_{t_{2}}^{1}\left|G\left(t_{1}, s\right)-G\left(t_{2}, s\right)\right| d s\right),
\end{gathered}
$$

that implies

$$
\begin{aligned}
& \left|T u\left(t_{1}\right)-T u\left(t_{2}\right)\right| \\
\leq & \frac{C^{\prime}}{\Gamma(q)} \int_{0}^{t_{1}}\left(t_{2}-s\right)^{q-1}-\left(t_{1}-s\right)^{q-1} \\
& +\left(t_{2}-t_{1}\right)\left((1-s)^{q-1}+q(q-1)\left(1+t_{2}^{2}+t_{1}^{2}+t_{1} t_{2}\right)(1-s)^{q-3}\right) d s \\
& +\int_{t_{1}}^{t_{2}}\left(t_{2}-s\right)^{q-1} \\
& +\left(t_{2}-t_{1}\right)\left((1-s)^{q-1}+q(q-1)\left(1+t_{2}^{2}+t_{1}^{2}+t_{1} t_{2}\right)(1-s)^{q-3}\right) d s \\
& +\int_{t_{2}}^{1}\left(t_{2}-t_{1}\right)\left((1-s)^{q-1}+q(q-1)\left(1+t_{2}^{2}+t_{1}^{2}+t_{1} t_{2}\right)(1-s)^{q-3}\right) d s
\end{aligned}
$$

if we consider the function $\Phi(x)=x^{q-1}-(q-1) x$, we see that $\Phi$ is decreasing on $[0,1]$, consequently 
$\left(t_{2}-s\right)^{q-1}-\left(t_{1}-s\right)^{q-1} \leq(q-1)\left(t_{2}-t_{1}\right)$, from this and using some elementary computations we deduce

$$
\left|T u\left(t_{1}\right)-T u\left(t_{2}\right)\right| \leq \frac{C^{\prime}\left(t_{2}-t_{1}\right)\left(102+49\left(t_{2}-t_{1}\right)\right)}{\Gamma(q)}
$$

On the other hand we have

$$
\begin{aligned}
& \left|{ }^{c} D_{0^{+}}^{\sigma} T u\left(t_{1}\right)-{ }^{c} D_{0^{+}}^{\sigma} T u\left(t_{2}\right)\right| \\
\leq & +\frac{1}{\Gamma(2-\sigma)} \int_{0}^{t_{1}}\left(\left(t_{1}-s\right)^{-\sigma+1}-\left(t_{2}-s\right)^{-\sigma+1}\right)\left|(T u(s))^{\prime \prime}\right| d s \\
& +\frac{1}{\Gamma(2-\sigma)} \int_{t_{1}}^{t_{2}}\left(t_{2}-s\right)^{-\sigma+1}\left|(T u(s))^{\prime \prime}\right| d s .
\end{aligned}
$$

Using (3.5) and (3.12) it yields

$$
\left|(T u(t))^{\prime \prime}\right| \leq[\psi(r)+\phi(r)+1] C_{2}^{\prime}
$$

then

$$
\begin{gathered}
\left|{ }^{c} D_{0^{+}}^{\sigma} T u\left(t_{1}\right)-{ }^{c} D_{0^{+}}^{\sigma} T u\left(t_{2}\right)\right| \leq \\
\frac{[\psi(r)+\phi(r)+1] C_{2}^{\prime}}{(2-\sigma) \Gamma(2-\sigma)}\left[2\left(t_{2}-t_{1}\right)^{2-\sigma}+t_{2}^{2-\sigma}-t_{1}^{2-\sigma}\right]
\end{gathered}
$$

when $t_{1} \rightarrow t_{2}$, in (3.14) and (3.16) then $\left|T u\left(t_{1}\right)-T u\left(t_{2}\right)\right|$ and $\left|{ }^{c} D_{0^{+}}^{\sigma} T u\left(t_{1}\right)-{ }^{c} D_{0^{+}}^{\sigma} T u\left(t_{2}\right)\right|$ tend to 0 . Consequently $T\left(B_{r}\right)$ is equicontinuous. From Arzela-Ascoli Theorem we deduce that $T$ is completely continuous.

Now we apply Leray Schauder nonlinear alternative to prove that $T$ has at least one nontrivial solution in $E$.

Let $\Omega=\{u \in E:\|u\|<r\}$, for any $u \in \partial \Omega$, such that $u=\lambda T u, 0<\lambda<1$, we get with the help of $(3.11)$

$$
|u(t)|=\lambda|T u(t)| \leq|T u(t)| \leq \frac{C_{1}}{\Gamma(q)}[\psi(r)+\phi(r)+1] .
$$

It follows from $(3.13)$

$$
\left|{ }^{c} D_{0^{+}}^{\sigma} u(t)\right| \leq C_{2}(\psi(r)+\phi(r)+1) .
$$

From (3.17), (3.18) and (3.8) we deduce that

$$
\|u\| \leq(\psi(r)+\phi(r)+1)\left(\frac{C_{1}}{\Gamma(q)}+C_{2}\right)<r
$$

this contradicts the fact that $u \in \partial \Omega$. Lemma 3.5 allows us to conclude that the operator $T$ has a fixed point $u^{*} \in \bar{\Omega}$ and then the fractional boundary value problem (P1) has a nontrivial solution $u^{*} \in E$. The proof is complete.

Example 3.6. The fractional boundary value problem

$$
\left\{\begin{array}{c}
{ }^{c} D_{0^{+}}^{\frac{7}{2}} u=\left(\frac{t}{10}\right)^{3} u+\left(\frac{t-1}{20}\right)^{2} D_{0^{+}}^{\frac{5}{4}} u+(t+1), \quad 0<t<1, \\
u(0)=u(1)=u^{\prime \prime}(1)=u^{\prime \prime}(0)=0,
\end{array}\right.
$$

has a unique solution in $E$.

Proof. We have $f(t, x, y)=\left(\frac{t}{10}\right)^{3} x+\left(\frac{t-1}{20}\right)^{2} y+(t+1), 3<q=\frac{7}{2}<4, \sigma=\frac{5}{4}<2$ and

$$
|f(t, x, \bar{x})-f(t, y, \bar{y})| \leq\left(\frac{t}{10}\right)^{3}|x-\bar{x}|+\left(\frac{t-1}{20}\right)^{2}|y-\bar{y}|
$$


then $g(t)=\left(\frac{t}{10}\right)^{3}$ and $h(t)=\left(\frac{t-1}{20}\right)^{2}$. Some calculus give

$$
\begin{aligned}
\left\|I_{0^{+}}^{q-1} g\right\|_{L^{1}} & =3.2065 \times 10^{-6}, \quad I_{0^{+}}^{q} g(1)=3.2064 \times 10^{-6}, \\
I_{0^{+}}^{q-2} g(1) & =0.11463 \times 10^{-3}, \quad C_{g}=0.24208 \times 10^{-3}, \quad A_{g}=9.7155 \times 10^{-4} \\
\left\|I_{0^{+}}^{q-1} h\right\|_{L^{1}} & =5.4709 \times 10^{-4}, \quad I_{0^{+}}^{q} h(1)=5.4709 \times 10^{-4}, \\
\left\|I_{0^{+}}^{q-\sigma-1} g\right\|_{L^{1}} & =3.2457 \times 10^{-6},\left\|I_{0^{+}}^{q-\sigma-1} h\right\|_{L^{1}}=5.1918 \times 10^{-4} \\
I_{0^{+}}^{q-2} h(1) & =0.32239 \times 10^{-2}, \quad C_{h}=0.21591 \times 10^{-2}, \quad A_{h}=2.8815 \times 10^{-2} \\
C_{g}+C_{h} & =0.45799 \times 10^{-2}<\frac{1}{2} \\
A_{g}+A_{h} & =2.5982 \times 10^{-3}<\frac{1}{2}
\end{aligned}
$$

Thus Theorem 3.2 implies that the fractional boundary value problem (3.19) has a unique in $E$.

Example 3.7. The fractional boundary value problem

$$
\left\{\begin{array}{c}
{ }^{c} D_{0^{+}}^{\frac{10}{3}} u=\frac{\left(1+t^{2}\right)}{100}\left(e^{-t} u^{2}+\ln \left(2+\left({ }^{c} D_{0^{+}}^{\frac{6}{5}} u\right)^{2}\right)+\frac{1}{1+t}\right)=0, \quad 0<t<1 \\
u(0)=u(1)=u^{\prime \prime}(1)=u^{\prime \prime}(0)=0
\end{array}\right.
$$

has at least one nontrivial solution in E. Applying Theorem 3.4, we have $q=\frac{10}{3}, \sigma=\frac{6}{5}$ and

$$
\begin{aligned}
|f(t, x, \bar{x})| & =\left(1+t^{2}\right) e^{-t}\left(\frac{x}{10}\right)^{2}+\left(1+t^{2}\right) \frac{\ln \left(2+\bar{x}^{2}\right)}{100}+\frac{\left(1+t^{2}\right)}{100(1+t)} \\
& \leq\left(\frac{|x|}{10}\right)^{2}\left(1+t^{2}\right)+\left(1+t^{2}\right) \frac{\ln \left(2+\bar{x}^{2}\right)}{100}+\left(1+t^{2}\right) \\
& \leq k(t) \psi(|x|)+h(t) \phi(|\bar{x}|)+g(t),
\end{aligned}
$$

where $k(t)=h(t)=g(t)=1+t^{2}, \psi(x)=\left(\frac{|x|}{10}\right)^{2}, \phi(\bar{x})=\frac{\ln \left(2+\bar{x}^{2}\right)}{100}, f(t, 0,0) \neq 0$.

Let us find $r$ such that (3.8) holds, for this we have

$$
\begin{aligned}
\left\|I_{0^{+}}^{q-1} g\right\|_{L^{1}}= & 0.117332, \quad I_{0^{+}}^{q-2} g(1)=1.0559, \quad I_{0^{+}}^{q-1} g(1)=0.40979 \\
\left\|I_{0^{+}}^{q-\sigma-1} g\right\|_{L^{1}}= & 0.50863, I_{0^{+}}^{q} g(1)=0.11733, \\
& 0.50863+\frac{1}{\Gamma\left(4-\frac{6}{5}\right)} 1.0559 \\
C_{1}= & 0.6453, \quad A_{g}=C_{2}=1.1385
\end{aligned}
$$

We see that (3.8) is equivalent to $1.3708\left(\left(\frac{r}{10}\right)^{2}+\frac{\ln \left(2+r^{2}\right)}{100}+1\right)-r$ which is negative for $r \geq 2$.

\section{References}

[1] G. A. Anastassiou, On right fractional calculus, Chaos, Solitons and Fractals, 42 (2009), 365-376.

[2] C.Z. Bai, Triple positive solutions for a boundary value problem of nonlinear fractional differential equation, Electron. J. Qual. Theory Diff. Equ. 24 (2008), 1-10.

[3] R. L. Bagley, A theoretical basis for the application of fractional calculus to viscoelasticity, Journal of Rheology, 27 (3) (1983), 201-210.

[4] K. Deimling, Nonlinear functional analysis, Springer, Berlin, 1985.

[5] R. Hilfer, Application of Fractional Calculus in Physics, World Scientific Singapur (2000), 699-707.

[6] H. Jafari, V. Daftardar-Gejji, Positive solutions of nonlinear fractional boundary value problems using adomian decomposition method, Appl. Math. Comput. 180 (2006), 700-706. 
[7] A.A. Kilbas, H.M. Srivastava, J.J. Trujillo, in: Jan van Mill (Ed.), Theory and Applications of Fractional Differential Equations, in: North-Holland Mathematics Studies, 204, Elsevier, Amsterdam, The Netherlands, 2006.

[8] S. Liang, J. Zhang, Existence and uniqueness of positive solutions to m-point boundary value problem for nonlinear fractional differential equation, J Appl Math Comput, DOI 10.1007/s12190-011-0475-2.

[9] S. Liang, J. Zhang, Positive solutions for boundary value problems of nonlinear fractional differential equation, Nonlinear Analysis, 71 (2009), 5545- 5550

[10] K. B. Oldham, Fractional differential equations in electrochemistry, Advances in Engineering Software (2009).

[11] I. Podlubny, Fractional Differential Equations, Mathematics in Science and Engineering, Vol. 1998, Academic Press, New York, London, Toronto, 1999.

[12] T. Qiu, Z. Bai, Existence of positive solutions for singular fractional equations, Electron. J. Diff. Equat. 146 (2008), 1-9.

[13] X. Su and L. Liu, Existence of solution for boundary value problem of nonlinear fractional differential equation, Appl. Math. J. Chinese Univ. Ser. B, 22 (3) (2007), 291-298.

[14] X. Xu, D. Jiang, C. Yuan, Multiple positive solutions for the boundary value problem of a nonlinear fractional differential equation, Nonlinear Anal, 71 (2009), 4676-4688.

[15] Y. Zhao, S. Sun, Z. Han, M. Zhang, Positive solutions for boundary value problems of nonlinear fractional differential equations, Applied Mathematics and Computation, 217 (2011), 6950-6958.

[16] Y. Zhao, S. Sun, Z. Han, Q. Li, The existence of multiple positive solutions for boundary value problems of nonlinear fractional differential equations, Commun. Nonlinear Sci. Numer. Simul. 16 (2011), 2086-2097. 